\title{
RESEÑA
}

\section{Enrique Pastor Seller. Trabajo Social con Comunidades / Social work with communities 2015. Madrid: Universitas}

Reseña realizada por José Javier Navarro Pérez

DOI: http://dx.doi.org/10.5944/comunitania.11.12

\section{Reseña:}

El Trabajo Social está de enhorabuena con la presente publicación. Un material que viene evolucionado a partir de la experiencia con Comunidades de Enrique Pastor Seller, Doctor en Sociología y Profesor Titular de Universidad adscrito al Departamento de Sociología y Trabajo Social de la Universidad de Murcia. Un material que aporta desde la esencia metodológica del Trabajo Social, una mirada aplicada en la intervención con comunidades. Se trata de un manual que aúna teoría y práctica, y que recupera el interés por movimientos evolucionados a partir de la articulación de engranajes colectivos.

Durante décadas, el desarrollo del trabajo social comunitario ha caracterizado el quehacer cotidiano de trabajadores/as sociales. Profesionales que han empoderado a las comunidades y que se han sumergido en el barro para orientar las estructuras en clave de desarrollo. La presente obra se construye a partir de orientaciones teóricas y habilidades prácticas, incidiendo en la naturaleza ideológica, en el saber hacer del trabajo social y la práctica comunitaria. Es una obra que tiene un público altamente transversal; desde profesores universitarios, investigadores, agentes de desarrollo comunitario, profesionales del desarrollo local, de planificación estratégica, formadores, hasta estudiantes de diferentes titulaciones académicas y doctorandos que deseen profundizar en las raíces de la comunidad y en las posibilidades de cambio y crecimiento que en la interacción con ella se generan.

Nos hallamos en un momento de pausa, amenazado por crisis ideológicas, y por la pasividad de las políticas públicas ante los problemas colectivos. El trabajo pone énfasis en la esencia y el compromiso ante la canalización de redes que permitan la retroalimentación y el crecimiento de las comunidades, en favor del desarrollo colectivo. Esta obra, contiene toda una declaración de intenciones en clave Freiriana: 
acciones conjuntas, fusionadas y dinámicas favorecedoras de una ciudadanía crítica como ente de transformación social. Sin duda, una obra de cabecera.

Un atractivo material de poco más de doscientas cincuenta interesantes páginas, organizado en siete capítulos. En ellos se analiza desde una perspectiva vanguardista los principales retos y futuro del trabajo social con comunidades. El autor se detiene en el capítulo primero en el análisis de los actuales contextos de acción transformación comunitaria enTrabajo Social: Ios desafíos del nuevo escenario local, las nuevas dimensiones que encierra el concepto urbano, las particularidades del escenario rural, además de los distintivos que engloba el escenario rural. El autor rompe con las clásicas etiquetas (individual vs. comunitario, macro vs. micro, rural vs. urbano, etc.) para adentrarse en un discurso actual del lenguaje comunitario. Se constata la importancia del desarrollo local como parte inherente a la proyección comunitaria, en un marco de sociedades globales.

El capítulo dos nos invita a adentrarnos en esta dimensión integradora; a caballo "entre lo estructural y lo relacional". El autor aborda la dimensión del trabajo social en y con la comunidad, penetrando en las distintas sensibilidades y redes que la configuran.

El capítulo tercero se adentra en los modelos teóricos del trabajo social con comunidades. Ofreciendo además una perspectiva introspectiva sobre teórico y el instrumental, que ha caracterizado al Trabajo Social en tiempos pretéritos. Forman parte de esta estructura, desde los modelos surgidos al calor del movimiento de reconceptualización y la participación y desarrollo de minorías, pasando por modelos de corte humanista y paradigmas ecológicos aplicados que tratan de corregir la inadaptación social, desechando los procesos de individualización y apostando rotundamente por el entorno cotidiano.

Seguidamente, el cuarto capítulo nos presenta las estrategias, apuestas y actuales retos que representan las potencialidades de la participación en los procesos de toma de decisiones, que al fin y al cabo infieren una elevada tasa de éxito en los procesos de planificación y desarrollo territorial. Desde esta panorámica se concibe a la comunidad como contexto, sistema y proceso para el cambio y la promoción social. Más adelante, el capítulo cinco el autor nos deja algunos detalles sobre el compromiso adquirido por el trabajo social del Siglo XXI con el desarrollo social local integral, tomando en consideración la construcción estratégica de identidades colectivas. Aparece como referente principal de las políticas sociales locales la democratización de los procesos participativos. Se establece como un estandarte de la dimensión local, y como clave en los procesos de crecimiento comunitario. Asimismo se presenta una hoja de ruta a partir de leyes autonómicas de segunda generación, donde se aprecian mecanismos de participación en el marco de las actuales políticas de bienestar. 
La obra se adentra en un capítulo sexto que aborda el proceso del Trabajo Social con comunidades, mostrando el interés que reúne la estrategia para este tipo de propuestas de intervención. Así, el autor se hace eco de las principales técnicas aplicadas al Trabajo Social Comunitario en el contexto actual y otras centradas en la participación de los sujetos de la comunidad, como herramienta para la transformación de la vida cotidiana en los escenarios comunes. El autor destaca la importancia que requiere implementar distintas visiones y enfoques teórico - procesuales para alcanzar objetivos centrados en la tarea.

El último capítulo se nos muestra la visión del Trabajo Social en Comunidades en su abordaje desde distintos sistemas de bienestar: los servicios sociales, la mediación con comunidades, la salud, la educación, la justicia, vivienda y la dependencia. Se analizan distintas experiencias de intervención comunitaria en diferentes áreas de nuestro entorno; se reflexiona sobre el interés por la sistematización y la redefinición de prácticas basadas en la evidencia que permitan una clara apuesta por prácticas comunitarias basadas en la excelencia.

En definitiva, la obra trata de posicionar al Trabajo Social como eje de las dinámicas comunitarias. Ofrece estrategias disponibles para profesionales del S. XXI que centran su práctica en la dinamización y participación de colectivos, incluyendo la creación de redes, la incidencia política e ideológica. Se trata de un material unívoco y que además se venía reclamando dada la complejidad de los nuevos escenarios sociales; un manual necesario para el posicionamiento de la disciplina del Trabajo Social, que alimenta la necesidad de sentir la calle desde el marco local e irrumpe con frescura ideológica en las individualidades subjetivas que dominan la escena cotidiana. 


\section{comunitania}

REVISTA INTERNACIONAL DE TRABAJO SOCIAL Y CIENCIAS SOCIALES

INTERNATIONAL JOURNAL OF SOCIAL WORK AND SOCIAL SCIENCES

\section{ARTICULOS/ARTIILLS}

El arte urbano como instrumento de empoderamiento y visibilización. El Festival de Asalto/

Urban art as an instrument of empowerment and visibility. Asalto festival

Cecilia Serrano-Martínez.

Págs 9-26

¿Usuarios o ciudadanos? Intervención y participación en las políticas y servicios sociales

/ Users or citizens? Intervention and participation in social politics and services

Miguel Arenas Martínez.

Págs 27-43

La violencia de género en las historias de vida de las mujeres que inician su trayectoria vital en la situación de vulnerabilidad / Gender-based violence in the life stories of women who begin their life trajectories in situations of vulnerability

Gabriela Moriana Mateo

Págs 45-67

Las consecuencias de la burocratización en las organizaciones de servicios sociales / The consequences of bureaucratizacion in the social service organizations

Xavier Montagud Mayor.

Págs 69-89

Trabajadores Sociales en Israel: protagonistas y testigos del cambio social en la población árabe Análisis de grupos de discusión y entrevistas a profesionales de los servicios sociales en Galilea y Haifa / Social Workers in Israel: protagonists and witnesses of social change in the Arab population. Analysis of focus groups and interviews with professionals in the personal social services in Galilee and Haifa

Alfredo Hidalgo Lavié, Javier Simonovich y Mirtha Ben-Nun

Págs 91-111

Software educativo Historits 1.0: Una propuesta innovadora para enseñar y aprender la historia del Trabajo Social / Educational software Historits 1.0: an innovative offer to teach and to learn the history of the Social Work

Eloy Casique Rojas y Yocelyn Castro.

Págs 113-139

"Lauream Pacis": Una Cultura de Paz a través del deporte / "Lauream Pacis": Peace Culture trough the Sports

Paris Alejandro Cabello Tijerina y Lina Guadalupe Sierra García

Págs 141-155

Medicina indígena tradicional, su enlace con la psiquiatría y la salud Pública / Traditional native medicine, its linkage with psychiatry and public health

Iván Noé Martínez-Salazar, Erika Acevedo-Stefanoni y Luis Angel Martínez-Hernández.

Págs 157-168

\section{BESEEIAAS/REVIEWS}

Antonio López Peláez. Teoría del Trabajo Social con Grupos. Segunda Edición revisada y ampliada /Theory of Social Work with Groups. 2 ed.

(por Emilio Díaz de Mera).....

Págs 169-174

Roberta Teresa Di Rosa. Mediación, Ciudadanía y Convivencia entre Culturas / Mediation,

Citizenship and Coexistence among Cultures

(por Alberto José Olalde Altarejos).

Págs 175-177

Maria Antonietta Selvaggio (a cura di), Educatrici di società. Racconti di donne e di cura / Educadores de la sociedad. Relatos de mujeres y del cuidado 2014

(por Fiorenza Deriu)

Págs 179-182

Enrique Pastor Seller. Trabajo Social con Comunidades / Social work with communities 2015 (por José Javier Navarro Pérez).

Págs 183-185

Enrique Pastor Seller, Gerardo Támez González y Karla Annett Cynthia Sáenz López.

Gobernabilidad, ciudadanía y democracia participativa. Análisis comparado España México /

Governance, citizenship and participatory democracy 2014. A comparative analysis between

Spain and Mexico. Madrid: Dykinson

(por Juan Bautista Martínez Fernández)

Págs 187-192

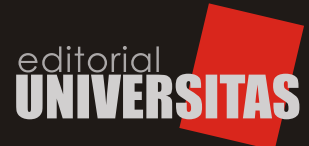

